\title{
The Potential Roles of Gamma-Glutamyltransferase Activity in the Pro- gression of Atherosclerosis and Cardiovascular Diseases
}

\author{
Aldo Paolicchi, Maria Franzini, Michele Emdin ${ }^{2}$, Claudio Passino ${ }^{2}$ and Alfonso Pompella ${ }^{1} * *$ \\ ${ }^{1}$ Department of Experimental Pathology, University of Pisa Medical School, and ${ }^{2}$ Institute of Clinical Physiology, Na- \\ tional Research Council-CNR, Pisa, Italy
}

\begin{abstract}
The oxidation of low density lipoproteins (LDL) is regarded as a critical factor in the pathogenesis of atherosclerosis, especially the initial steps of the disease. In addition, other oxidative events have been shown to participate in the progression of atherosclerosis and precipitation of cardiovascular events, through modulation of important components of lesions of the vessel wall (smooth muscle cell proliferation, protease/antiprotease balance, endothelial functions). Our recent studies have provided evidence that the enzyme gamma-glutamyltransferase (GGT), normally found in serum, is often accumulated within the plaque environment in substantial amounts, and that this activity is a potential source of a variety of prooxidant species. Concurrently, epidemiological research has conclusively documented that the serum levels of GGT are an independent factor in prognosis of myocardial infarction and stroke in atherosclerotic patients. Several signs suggest that the GGT appearing in plaque tissue may originate from the serum enzyme, which in facts associates with the circulating lipoprotein fractions. Thus, data seem to point out that pathogenesis of atherosclerosis - and in particular of the events leading to progression of the disease and acute cardiovascular events - might include an as yet unexplored pathway, based on the prooxidant effects of gamma-glutamyltransferase accumulating as a result of LDL entry in the vessel wall.
\end{abstract}

Keywords: Serum $\gamma$-glutamyltransferase, atherosclerosis, atherosclerotic plaque, oxidative stress, glutathione, protein oxidation.

\section{INTRODUCTION}

In dealing with atherosclerotic patients, much attention is devoted to predicting which subjects will experience acute, potentially fatal cardiovascular events. The focus is therefore on the numerous factors capable of affecting plaque stability, i.e. the pathophysiological, cellular and biochemical determinants that have been shown to participate in the progression of atherosclerotic lesions. The understanding of these factors would in fact allow the identification of high-risk lesions, in which the pathological changes produced by the disease process are more likely to result in plaque rupture, thrombosis and the associated ischemic/necrotic sequelae; establishing timely treatment for such lesions would clearly represent a major advance in secondary prevention and therapy.

Oxidative reactions occupy an important position among factors of plaque progression and "destabilization" [1]. One specific oxidative event - the oxidation of low density lipoprotein (LDL) - had been highlighted by earlier studies as the single crucial event in pathogenesis of atherosclerosis, with special reference to the initiation of lesions. Subsequent research has shown however that the implication of oxidative processes may be considerably wider, extending to include effects on several other important pathological changes occurring in the diseased vessel wall. Thus, it has been

*Address correspondence to this author at the Dipartim. di Patologia Sperimentale BMIE, Scuola Medica, Università di Pisa, Via Roma 55, 56126 Pisa, Italy; Tel: +390502218537 ; Fax: +390502218537 ;

E-mail: apompella@biomed.unipi.it shown that oxidants and redox reactions are involved in stimulation of smooth muscle cell proliferation, contributing to lesion thickness and expansion. The activities of matrix metalloproteinases and of their inhibitors - major determinants of plaque stability - are modulated through redox changes in critical portions of their protein structure. Some aspects of endothelial dysfunction [e.g. impairment of nitric oxide (NO) production, expression patterns and levels of adhesion molecules] are also affected by oxidative events, and several other redox-sensitive steps are present in signal transduction, apoptotic cell death and platelet function.

Altogether, current experimental studies consistently indicate that oxidation/reduction processes may be frequently involved in the production of changes capable of turning a mildly stenotic, silent plaque into a vulnerable, symptomatic and potentially fatal lesion. A series of enzyme activities, expressed by cellular elements present in the lesions (endothelium, phagocytes, smooth muscle cells, fibroblasts) have been proposed as sources of the oxidants involved; these include NADPH-oxidases, NO-synthase, xantine oxidase, myeloperoxidase, lipoxygenases and enzymes of mitochondrial respiration. Non-enzymic prooxidants also participate in the reactions, such as transition metals (iron, copper) and lipid peroxides. However, the relative roles and importance of each of the mentioned factors in the "oxidative progression" of lesions have not been elucidated yet, and are likely to depend on which aspect of the process is being considered; the whole matter is in fact the subject for extensive experimental research.

(c) 2006 Bentham Science Publishers Ltd. 


\section{$\gamma$-GLUTAMYLTRANSFERASE (GGT) ACTIVITY: A NOVEL MARKER OF ATHEROSCLEROSIS}

Although best known as a reliable index of hepatobiliary dysfunction and alcohol abuse [2], serum GGT levels have been repeatedly proposed to play an independent role in clinical evolution of cardiovascular diseases related with atherosclerosis [3]. Several studies had documented that serum GGT is associated with overall mortality and cardiovascular events in both unselected populations [4-7] and patients with ascertained coronary artery disease, independent of all confounders including liver function and alcohol consumption [8]. A recent prospective study, carried out in as many as 163,944 adult subjects [9], has now rather conclusively shown that serum GGT levels are an independent prognostic factor for fatal events of chronic forms of coronary artery disease, congestive heart failure and ischemic or hemorrhagic stroke. The finding was true for both sexes, with a clear dose-response relationship and a stronger significance as far as younger subjects were concerned.

The prognostic value of serum GGT activity for cardiac death and non-fatal infarction had been previously documented in a prospective study of our own, including a sixyear follow-up of 469 patients with ischemic syndrome and angiographically documented coronary artery disease [10]. In this study, the significance of serum GGT was more evident in a subset of patients prone to plaque complications, i.e. characterized by the association of diffuse atherosclerosis (multivessel disease) and a history of previous myocardial infarction (approx. 36\% of the whole population). The risk was increasing using two different GGT cut-off values ( 25 or $40 \mathrm{U} / \mathrm{l}$, both generally considered within the normal range), the event excess being concentrated within the first threeyear period. The prognostic significance of serum GGT was thus correlated with the diffusion of coronary artery disease, and interestingly, the significance of serum GGT appeared to depend on the instability of plaques, as indicated by the fact that the prognostic value of GGT disappeared after revascularization by angioplasty, a procedure thought to effect plaque stabilization. The unfavourable prognosis signaled by elevated serum GGT appeared thus to apply specifically to patients with vulnerable plaques, suggesting that connections of some kind may exist between GGT and the processes involved in plaque stability [10].

It should be stressed that all of the studies mentioned above refer to "normal" serum GGT, i.e. values within the laboratory reference range, such as in the large prospective study by Wannamethee et al. (7613 middle-aged men, with a 11-years follow-up), showing that GGT levels in the normal range (top quintile of distribution) were strongly associated with a significant increase in deaths from ischemic heart disease [6]. Serum GGT was positively correlated with preexisting ischemic heart disease (IHD), diabetes mellitus, antihypertensive medication, systolic and diastolic blood pressure, total and high density lipoprotein (HDL) cholesterol, heart rate and blood glucose. After adjustment for these variables, elevated GGT (highest quintile, $\geq 24 \mathrm{U} / \mathrm{l}$, vs. the rest) was still associated with a significant increase in mortality from all causes and from IHD. The increased risk of IHD mortality was more marked for patients with evidence of IHD at screening, particularly those with previous myo- cardial infarction [6]. The increased mortality in men without heart disease was obviously dependent on other causes; on the other hand, the findings in ischemic patients again pointed to a connection of serum GGT with unknown aspects of the underlying atherosclerotic coronary artery disease.

While the clinical studies mentioned above suggest an implication of circulating GGT in the evolution of disease, an indirect confirmation derives from additional evidence obtained by histochemistry. Histochemical studies performed in our laboratories showed that intense GGT activity is often present in the intimal layers of human atherosclerotic lesions, where it is apparently expressed in $\mathrm{CD}^{+} 8^{+}$macrophage-derived foam cells [11, 12]. GGT-positive foam cells were found to co-localize with immunoreactive oxidized LDL [13], and catalytically active GGT could also be detected in microthrombi adhering to the surface of atheromas [14]. Whether GGT activity found in plaque tissue is actually participating in the disease - or is rather an epiphenomenon lacking specific significance - remains to be ascertained; at least two questions arise: i) how could GGT activity affect the progression of the disease, and ii) how are serum levels of GGT related with the enzyme detectable in plaques?

\section{FUNCTIONS OF $\gamma$-GLUTAMYLTRANSFERASE ACTIVITY - A DOUBLE-EDGED SWORD}

The biochemical functions of GGT enzyme activity are complex, and its overall physiological significance has not been fully appreciated yet. Several aspects of GGT activity are indeed capable of promoting effects of oxidative nature, which in principle might contribute to progression of the atherosclerotic process. Most of the knowledge about the functions of GGT is concerned with the roles played by this activity at the cellular level. High GGT levels are expressed in kidney tubules, biliary epithelium and brain capillaries; however, lower or trace levels are detectable in many more cell types, e.g. blood cells, endothelium, and notably secretory and absorptive cells [15]. Being located at the plasma membrane level, GGT catalyzes the first step in the degradation of extracellular glutathione (GSH), i.e. the hydrolysis of the gamma-glutamyl bond between glutamate and cysteine. In this process GGT releases the dipeptide cysteinyl-glycine, which is subsequently cleaved to cysteine and glycine by plasma membrane dipeptidase activities; thus, GGT activity provides cells first of all with a means for the recovery of precursors needed to reconstitute the intracellular levels of glutathione, the main cellular antioxidant (Fig. 1A). Novel insights however have been prompted by studies showing the ability of GGT to play a prooxidant role under selected conditions. The key issue in this alternative function of GGT is related to the reducing properties of GSH and the thiol metabolites originating from GGT activity. Thiols - especially when dissociated to their thiolate anion forms $\left(\mathrm{R}-\mathrm{S}^{-}\right)$- can effect the reduction of metal cations, e.g. iron and copper. Electrons can then be transferred in turn from metal ions to molecular oxygen, thus generating the reactive oxygen species superoxide anion and hydrogen peroxide, both capable of stimulating prooxidant reactions. The sequence - a true "redox cycling" of metals [16] - can proceed with even minimal concentrations of metal ions as long as the electron donors (thiols) and electron acceptor (molecular oxygen) are 
available. In this way, and paradoxically, the 'reducing' abilities of thiols are eventually turned into overall 'oxidizing' effects. Stark et al. [17] were the first to propose that the catabolism of GSH can play a prooxidant role under selected conditions. These authors suggested that the GGT-mediated cleavage of GSH - allegedly through the generation of the more reactive thiol cysteinyl-glycine - could cause the reduction of ferric iron $\mathrm{Fe}(\mathrm{III})$ to ferrous iron $\mathrm{Fe}(\mathrm{II})$, thus starting an iron-dependent redox-cycling process liable to result in the production of reactive oxygen species (ROS) and the stimulation of oxidative reactions (Fig. 1B). Subsequent studies from our and other laboratories have repeatedly documented the production of ROS during the catabolism of GSH mediated by cellular GGT. The biochemical processes involved have been characterized (reviewed in [14]), and some of the redox effects occurring at the cellular level have been described, e.g. on the activation status of transcription factors and apoptotic signalling pathways [14, 18, 19].
The observation of such prooxidant reactions has refined our understanding of GGT function, whose significance appears to be dual. At a cellular level, it is conceivable that the prooxidant effects of plasma membrane GGT are normally balanced by its established role in favouring the cellular uptake of precursors for GSH resynthesis, thus allowing the maintenance of cellular antioxidant defenses. On the other hand, the functions of soluble GGT activity in serum are less directly related to the cellular supply of GSH, and conditions likely exist in which its prooxidant effects may be prevailing. The free iron required for such processes can originate from iron storage proteins; it was in fact shown that GGT prooxidant effects are sustained by both transferrin and ferritin [14, 20]. The emerging prooxidant potential of serum GGT can help explain the recent observation that baseline serum GGT can predict future elevations of serum $\mathrm{F}_{2}$-isoprostanes, a sensitive marker of oxidative stress [21, 22]. As far as the plaque environment, free iron is present [23], at levels sufficient to sustain the GGT-mediated prooxidant effects [11].

\section{A}
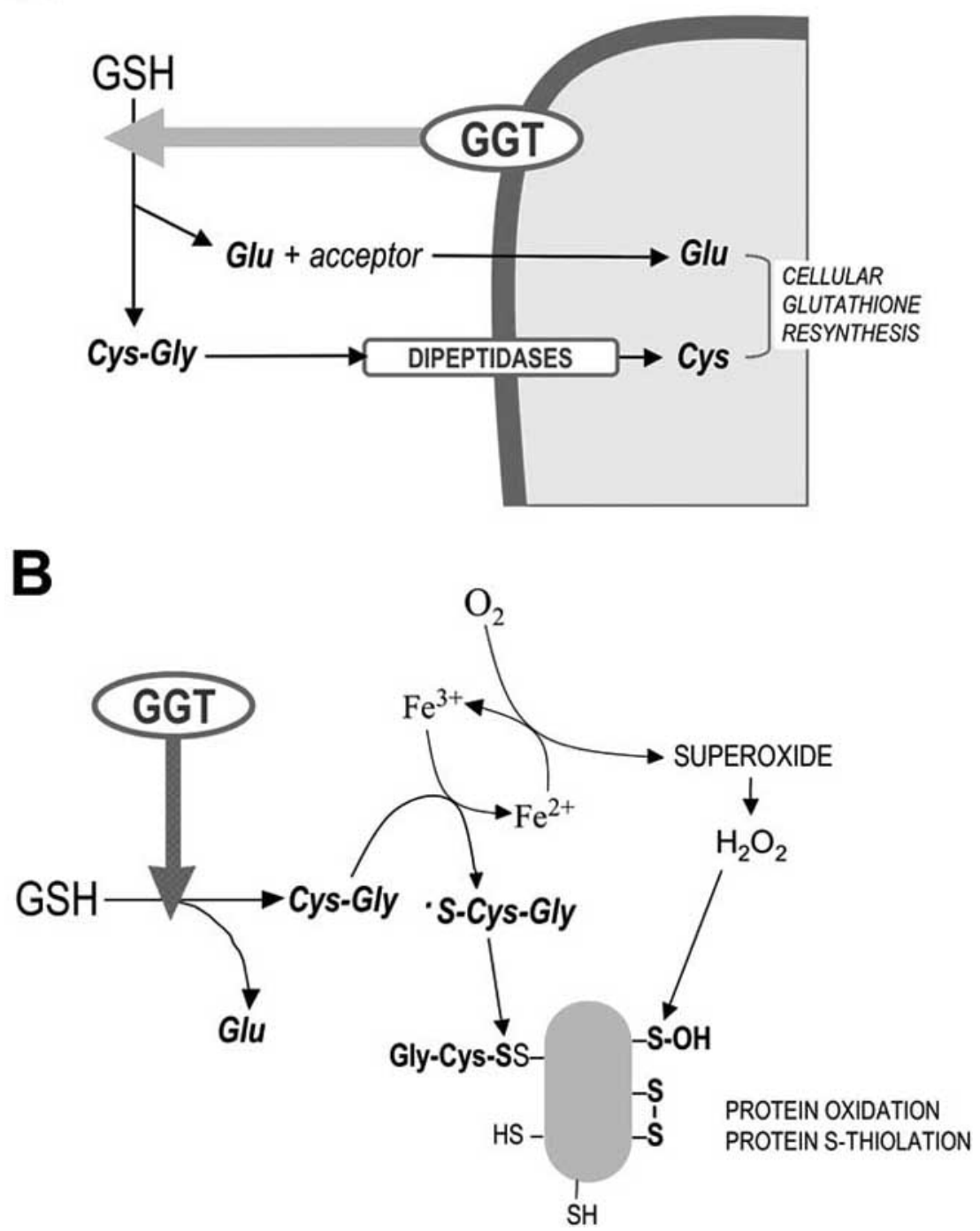

Fig. (1). Functions of $\gamma$-glutamyltransferase activity: (A) cellular supply of precursors for the resynthesis of intracellular stores of the antioxidant glutathione, and (B) promotion of metal-catalyzed redox reactions, leading to the appearance of reactive oxygen species, free radicals and oxidative modifications on proteins. $(G S H)$, glutathione; (Cys-Gly), cysteinyl-glycine. 


\section{CIRCULATING GGT VS. ENZYME ACTIVITY DETECTABLE IN PLAQUES}

The GGT enzyme activity circulating in serum is thought to be mainly released from the liver [1]. Constitutively high serum GGT has been reported in families as an inherited autosomic dominant character, and in the apparent absence of any disease (see e.g. [24]). Genetic effects could in principle involve either the release of GGT from liver cells or its clearance from circulation. Other liver enzymes partly share the same effects, and this seems to indicate that genetic factors may be operative at the level of GGT release in blood [25]. The importance of genetic factors is still a matter for discussion; current view is that a mixture of genetic and environmental factors can influence serum GGT $[25,26]$. Another line of evidence points to the possibility that the source of serum GGT may be identified in circulating platelets, as the two parameters are usually correlated [27]. Thus, it has been proposed that the increased GGT levels observed in atherosclerotic patients may originate from increased platelet consumption at the site of plaques [28].

Interestingly, serum GGT is partially adsorbed onto circulating LDL [29, 30]. Details of such association were recently investigated by us in a population of patients with angiographically documented coronary artery disease (CAD) [31]. As GGT activity in non-cholestatic patients is mainly associated with HDL and LDL [30], the amount of GGT activity associated with polycation-precipitated $\beta$ lipoproteins $(\beta-\mathrm{LP})$ - which include LDL, intermediate density lipoprotein (IDL) and very low density lipoprotein (VLDL), but not HDL - was assumed as an index of LDLassociated GGT. It was thus observed that both in controls and CAD patients the amount of GGT associated with $\beta$-LP is directly proportional to total serum GGT levels; in CAD patients however, the $\beta$-LP-associated fraction was significantly lower. Furthermore, serum GGT is known to increase with age, and such increase was indeed found in the control group; in contrast, significantly lower total serum GGT levels were detected in older CAD patients, though the ratio between $\beta$-LP-bound and total GGT remained constant [31]. It remains to be elucidated whether this phenomenon depends on the absence in CAD patients of the factor(s) eliciting the increase of serum GGT with age, or rather to a selective loss of patients with higher GGT [10], due to (GGTrelated?) complications of $\mathrm{CAD}$, i.e. cardiac death and stroke.

The reasons for the association of GGT with LDL likely lie in the peculiar structure of GGT, a dimeric protein whose heavy chain contains the lipophilic domain responsible for the plasma membrane insertion of the cellular enzyme. Several proteases are capable of digesting the lipophilic domain, thus producing a hydrophilic form of the protein [32]. The specific association of GGT with LDL and the nature of the protein were further confirmed by ultracentrifugation. We found that the LDL-associated enzyme consists in an entire heavy chain, indicating that the amphiphilic form of the enzyme is involved [31]. It is known however that the incubation of amphiphilic GGT with isolated lipoprotein does not produce GGT-lipoprotein complexes [33], suggesting that the interaction of GGT with lipoprotein may be different from the one with plasma membrane lipids. Indeed, affinity- adsorbed LDL released most of its GGT activity following extensive washing, indicating a rather labile LDL/GGT association [31].

As the $\beta$-LP-associated fraction of GGT is proportional to total serum GGT, the binding of GGT with LDL might itself be related to the reported increase of cardiovascular risk. What mechanisms could then account for the observed decrease of $\beta$-LP-bound GGT in CAD patients, and the decrease of total GGT levels in older ones? Conceivably, the reasons might be (i) a less efficient association of GGT with LDL in these patients, (ii) an enhanced release of GGT from LDL, or (iii) an enhanced uptake of LDL-GGT complexes within atherosclerotic lesions, possibly of those presenting with higher GGT contents. The latter hypothesis is particularly attractive, as it would allow the integration of an overall picture with the histochemical findings (Fig. 2): the enhanced uptake of LDL-GGT complexes, leading to the appearance of significant deposits of active GGT within the lesions, might be a discrete step in atherogenesis, potentially explaining the reported correlation between higher levels of serum GGT and the clinical consequences of atherosclerosis.

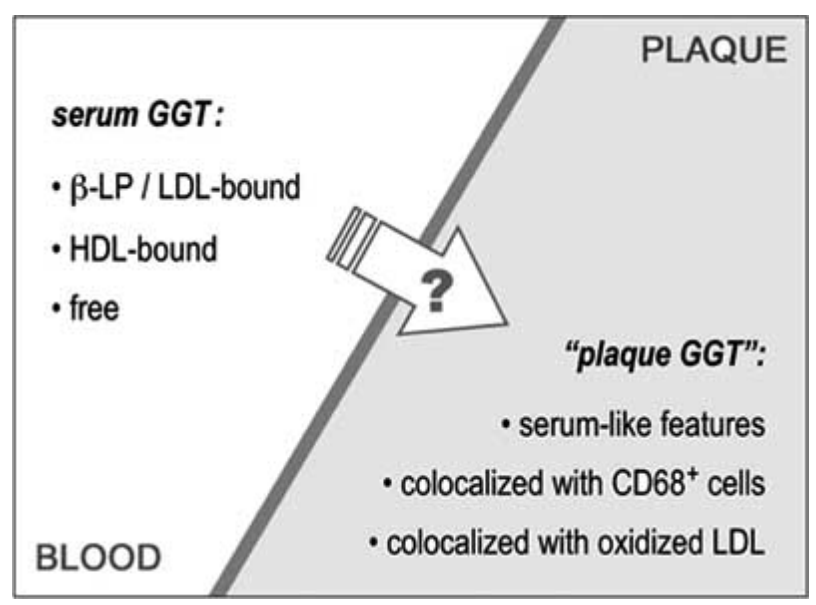

Fig. (2). The envisaged relationships between GGT activity circulating in serum in association with distinct lipoprotein fractions, and GGT activity detectable in atherosclerotic lesions. Available data suggest that the latter might result from the uptake of GGT/LDL complexes within the arterial wall.

\section{CONCLUDING REMARKS}

Studies in our laboratory are currently aimed to a further biochemical characterization of GGT present in human atherosclerotic plaques. As a confirmation of the hypothesis discussed above, preliminary data indicate that the enzyme protein accumulating in lesions has molecular weight and chromatographic properties compatible with those of serum GGT. In addition, proteins recovered form plaque tissue present with some degree of S-cysteyl-glycylation, i.e. the form of protein S-thiolation specifically catalysed by GGT [34], thus witnessing the occurrence of prooxidant reactions promoted by the accumulated enzyme [Franzini et al. manuscript in preparation]. It seems therefore reasonable to speculate that the prooxidant reactions promoted by GGT activity might contribute to the (oxidative) processes in- 
volved in several aspects of plaque destabilization. Further studies are necessary however in order to identify the factors that favour the accumulation of GGT within plaques, and possibly accelerate it in selected subjects.

\section{ACKNOWLEDGEMENTS}

Research described in the present paper was supported by the Italian Ministry for Education, University and Research (MIUR-FIRB and -PRIN Funds). Additional support was provided by the Fondazione per la Ricerca sulla Fibrosi Cistica (Verona, Italy).

\section{REFERENCES}

[1] Stocker R, Keaney JF Jr. Role of oxidative modifications in atherosclerosis. Physiol Rev 2004; 84: 1381-1478.

[2] Whitfield JB. Gamma glutamyl transferase. Crit Rev Clin Lab Sci 2001; 38: 263-355.

[3] Pompella A, Emdin M, Passino C, Paolicchi A: The significance of serum gamma-glutamyltransferase in cardiovascular diseases. Clin Chem Lab Med 2004; 42: 1085-91.

[4] Conigrave KM, Saunders JB, Reznik RB, Whitfield JB. Prediction of alcohol-related harm by laboratory test results. Clin Chem 1993; 39: 2266-70.

[5] Brenner H, Rothenbacher D, Arndt V, Schuberth S, Fraisse E, Fliedner TM. Distribution, determinants, and prognostic value of $\gamma$ glutamyltranspeptidase for all-cause mortality in a cohort of construction workers from south Germany. Prev Med 1997; 26: 30510 .

[6] Wannamethee G, Ebrahim S, Shaper AG. $\gamma$-Glutamyltransferase: determinants and association with mortality from ischemic heart disease and all causes. Am J Epidemiol 1995; 142: 699-708.

[7] Jousilahti P, Rastenyte D, Tuomilehto J. Serum $\gamma$ glutamyltransferase, self-reported alcohol drinking, and the risk of stroke. Stroke 2000; 31: 1851-55.

[8] Karlson BW, Wiklund O, Hallfren P, Sjolin M, Lindquist J, Herlitz J. Ten-year mortality among patients with a very small or unconfirmed acute myocardial infarction in relation to clinical history, metabolic screening and signs of myocardial ischemia. J Intern Med 2000; 247: 449-56.

[9] Ruttmann E, Brant LJ, Concin H, Diem G, Rapp K, Ulmer H. Vorarlberg Health Monitoring and Promotion Program Study Group. Gamma-glutamyltransferase as a risk factor for cardiovascular disease mortality: an epidemiological investigation in a cohort of 163,944 Austrian adults. Circulation 2005; 112: 2130-7.

[10] Emdin M, Passino C, Michelassi C, et al. Prognostic value of serum $\gamma$-glutamyl transferase activity after myocardial infarction. Eur Heart J 2001; 22: 1802-7.

[11] Paolicchi A, Minotti G, Tonarelli P, et al. $\gamma$-Glutamyl transpeptidase-dependent iron reduction and low density lipoprotein oxidation - a potential mechanism in atherosclerosis. J Invest Med 1999; 47: 151-60.

[12] Paolicchi A, Emdin E, Ghliozeni E, et al. Atherosclerotic plaques contain $\gamma$-glutamyl transpeptidase activity. Circulation 2004; 109: 1440.

[13] Emdin M, Passino M, Donato L, Paolicchi A, Pompella A. Serum $\gamma$-glutamyltransferase as a risk factor of ischemic stroke might be independent of alcohol consumption. Stroke 2002; 33: 1163-4.

[14] Dominici S, Paolicchi A, Lorenzini E, et al. $\gamma$-Glutamyltransferasedependent prooxidant reactions: a factor in multiple processes. BioFactors 2003; 17: 187-98.

[15] Hanigan MH, Frierson HF Jr. Immunohistochemical detection of gamma-glutamyl transpeptidase in normal human tissue. J Histochem Cytochem 1996; 44: 1101-8.
[16] Kappus H, SIes H. Toxic drug effects associated with oxygen metabolism: Redox cycling and lipid peroxidation. Experientia 1981; 37: 1233-41.

[17] Stark A-A, Zeiger E, Pagano DA. Glutathione metabolism by $\gamma$ glutamyl transpeptidase leads to lipid peroxidation: characterization of the system and relevance to hepatocarcinogenesis. Carcinogenesis 1993; 14: 183-89.

[18] Del Bello B, Paolicchi A, Comporti M, Pompella A, Maellaro E. Hydrogen peroxide produced during $\gamma$-glutamyl transpeptidase activity is involved in prevention of apoptosis and maintainance of proliferation in U937 cells. FASEB J 1999;13:69-79.

[19] Paolicchi A, Dominici S, Pieri L, Maellaro E, Pompella A. Glutathione catabolism as a signalling mechanism. Biochem Pharmacol 2002;64:1029-37.

[20] Corti A, Raggi C, Franzini M, Paolicchi A, Pompella A, Casini AF. Plasma membrane $\gamma$-glutamyltransferase activity facilitates the uptake of vitamin $\mathrm{C}$ in melanoma cells. Free Rad Biol Med 2005; 7 : 911-18.

[21] Lee DH, Jacobs DRJr, Gross M, Kiefe CI, Roseman J, Lewis CE, et al. $\gamma$-Glutamyltransferase is a predictor of incident diabetes and hypertension. Coronary Artery Risk Development in Young Adults (CARDIA) Study. Clin Chem 2003; 49: 1358-66.

[22] Lee DH, Blomhoff R, Jacobs DR. Is serum $\gamma$-glutamyltransferase a marker of oxidative stress? Free Rad Res 2004; 38: 535-9.

[23] Pang JH, Jiang MJ, Chen YL, Wang FW, Chu SH, Chan LY. Increased ferritin gene expression in atherosclerotic lesions. J Clin Invest 1996; 97: 2204-12.

[24] Bibas M, Zampa G, Procopio A, Guaitolini R. High serum $\gamma$ glutamyltransferase concentrations in a family. New Eng J Med 1994; 330: 1832-33.

[25] Whitfield JB, Zhu G, Nestler JE, Heath AC, Martin NG. Genetic covariation between serum $\gamma$-glutamyltransferase activity and cardiovascular risk factors. Clin Chem 2002; 48: 1426-31.

[26] Bathum L, Petersen HC, Rosholm J-U, et al. Evidence for a substantial genetic influence on biochemical liver function tests: results from a population-based Danish twin study. Clin Chem 2001; 47: 81-7.

[27] Bolodeoku JA, Ganotakis ES, Mikhailidis DP, Winder AF. Correlation between serum $\gamma$-glutamyltransferase activity and the platelet count. Platelets 1997; 8: 333-5.

[28] Krijgsman B, Hamilton G, Mikhailidis DP. $\gamma$-Glutamyltransferase and vascular disease. Clin Chem 2003; 49: 522-3.

[29] Huseby NE. Multiple forms of serum gamma-glutamyltransferase. Association of the enzyme with lipoproteins. Clin Chim Acta 1982; 124: 103-12.

[30] Watanabe M, Taketa K, Izumi M, Nagashima H. Association of gamma-glutamyltransferase with plasma lipoprotein and lipidprotein complex in cholestasis. Hepatogastroenterology 1984; 31 : 204-7.

[31] Paolicchi A, Emdin M, Passino C, Lorenzini E, Titta F, Marchi S, Malvaldi G, Pompella A. $\beta$-Lipoprotein- and LDL-associated serum $\gamma$-glutamyltransferase in patients with coronary atherosclerosis. Atherosclerosis 2005; in press.

[32] Meister A. In: Larsson A, Orrenius S, Holmgren A, Mannervik B Eds, Functions of glutathione: biochemical, toxicological and clinical aspects. New York, Raven Press 1983; 1-22.

[33] Artur Y, Wellman-Bednawska M, Jacquier A, Siest G. Associations between serum gammaglutamyltransferase and apolipoproteins: relationships with hepatobiliary disease. Clin Chem 1984; 30: 1318-21.

[34] Corti A, Paolicchi A, Franzini M, Dominici S, Casini AF, Pompella A. The S-thiolating activity of membrane $\gamma$-glutamyltransferase: formation of cysteinyl-glycine mixed disulfides with cellular proteins and in the cell microenvironment. Antiox Redox Signall 2005; 7: 911-8. 